\title{
Théologiques
}

\section{La pierre rejetée par les bâtisseurs}

\section{René Girard}

Volume 13, numéro 2, automne 2005

Violence et souffrance rédemptrices

URI : https://id.erudit.org/iderudit/013609ar

DOI : https://doi.org/10.7202/013609ar

Aller au sommaire du numéro

\section{Éditeur(s)}

Faculté de théologie et de sciences des religions, Université de Montréal

ISSN

1188-7109 (imprimé)

1492-1413 (numérique)

Découvrir la revue

\section{Citer cet article}

Girard, R. (2005). La pierre rejetée par les bâtisseurs. Théologiques, 13(2), 165-179. https://doi.org/10.7202/013609ar

\section{Résumé de l'article}

Après avoir précisé le sens premier de l'expression polysémique « bouc émissaire ", l'auteur démontre l'universalité du phénomène ainsi désigné, depuis les sociétés archaïques jusqu'aux contemporaines, universalité qui trouve son fondement dans l'existence d'une hiérarchie sacrificielle commune à l'humanité entière. Sur ce fond, l'auteur compare le judéo-christianisme aux religions archaïques, d'une part explicitant l'élément essentiel qu'ils partagent, le phénomène du bouc émissaire, d'autre part s'inscrivant en faux contre l'assimilation de l'expression judéo-chrétienne de ce phénomène à ses présumés parallèles rencontrés dans les religions archaïques. Colonne vertébrale du nihilisme moderne, cette assimilation simpliste occulte sur ce chapitre l'unicité du judéo-christianisme, qui seul défend et réhabilite ses boucs émissaires.
Tous droits réservés @ $\odot$ Faculté de théologie et de sciences des religions, Université de Montréal, 2006
Ce document est protégé par la loi sur le droit d'auteur. L'utilisation des services d'Érudit (y compris la reproduction) est assujettie à sa politique d'utilisation que vous pouvez consulter en ligne.

https://apropos.erudit.org/fr/usagers/politique-dutilisation/ 


\section{La pierre rejetée par les bâtisseurs}

René GiRARD

Professeur émérite

Stanford University

1. La signification de «bouc émissaire»

\subsection{La polysémie de l'expression}

L'expression «bouc émissaire» a trois significations qu'il importe de ne pas confondre. La première, la plus ancienne, traduit le terme hébreu qui, dans le chapitre 16 du Lévitique, désigne la victime d'un rite très ancien, le rite du bouc émissaire. Tous les ans, à Yom Kippour, le grand-prêtre posait les mains sur la tête d'un bouc. Ce geste était censé transférer à celui-ci tous les péchés de la communauté. Pour se débarrasser de ceux-ci, il ne restait plus qu'à chasser l'animal dans le désert, à faire de lui le bouc émissaire.

La seconde signification désigne tous les rites analogues à celui du Lévitique dans toutes les communautés humaines. Ce sont partout les mêmes efforts pour se défaire des désordres et des violences à l'intérieur de ces communautés, par l'intermédiaire de victimes animales ou humaines violemment expulsées ou massacrées. L'anthropologue qui le premier a repéré l'universalité de ces rites, c'est Frazer, dans Le rameau d'or (1890). Il leur a donné à tous la même étiquette: rites de bouc émissaire. Cette généralisation du rite hébreu est légitime dans la mesure où l'on n'en conclut pas, comme on le fait parfois, que ces rites ont quelque chose de spécifiquement biblique. Puisqu'ils se ressemblent tous, on peut recourir pour les désigner à n'importe lequel d'entre eux, et Frazer a choisi le plus connu. On pourrait aussi bien dire «rites de pharmakos", c'est-à-dire recourir au bouc émissaire grec plutôt qu'à l'hébreu. Si on ne le fait pas, c'est sans doute parce que le terme évoque quelque chose de plus sinistre 
que le bouc chassé dans le désert: le pharmakos était un être humain que les cités grecques massacraient rituellement lors des fêtes de Dionysos.

La troisième signification de "bouc émissaire» apparaît dans les langues occidentales au début de l'ère moderne. En français, comme en anglais, en italien, etc., on entend le plus souvent par «bouc émissaire» la victime d'une hostilité qui affecte une communauté entière contre un individu ou un groupe d'individus innocents, non-pertinents. Plus une communauté est perturbée, plus elle tend à décharger son angoisse contre des boucs émissaires dans ce troisième sens.

Frazer a fort bien vu les deux premiers sens, mais pas le troisième. Il n'a pas soupçonné que les phénomènes irrationnels de discrimination et de persécution, même dans l'Angleterre de son temps, pourraient avoir un rapport avec les rites archaïques. Il nie implicitement toute parenté entre notre troisième sens de «bouc émissaire" et les deux premiers. On comprend sans peine pourquoi. Bien que fort perspicace pour certaines choses, ce grand ethnologue est aussi très imbu de la supériorité occidentale et moderne, anglaise surtout. Il ne voit rien de commun, sous le rapport de la violence arbitraire, entre ceux qu'il traite constamment de rude savages et les sujets de sa très gracieuse majesté britannique.

C'est là un préjugé auquel il faut renoncer. La langue populaire a raison contre Frazer. Pour comprendre les religions archaïques, il faut relier sans les confondre les trois significations de «bouc émissaire». Bien que privés désormais de sanction religieuse, les phénomènes de violence collective se perpétuent parmi nous sous une forme plus ou moins clandestine, souvent affaiblie, mais susceptible de retrouver parfois une virulence démentielle, dans le nazisme par exemple.

\subsection{Le sens premier de "bouc émissaire»}

Les dictionnaires qualifient de "dérivé» le sens moderne de «bouc émissaire ", par opposition au bouc du Lévitique qui serait "le sens propre». L'animal rituel est premier, certes, du point de vue chronologique, mais pour comprendre sa raison d'être, le sens moderne, je pense, est essentiel. Le phénomène psychosocial est sous-jacent, je crois, à tous les phénomènes rituels. Il faut invertir la hiérarchie du dictionnaire et situer l'origine des rites dans une tendance universelle des communautés troublées à persécuter les êtres les plus faibles, les moins capables de riposter, notamment les minorités raciales ou religieuses. Nous percevons mieux cette tendance que 
nos ancêtres et c'est pourquoi nous n'auréolons plus nos boucs émissaires de religieux.

Pour comprendre les religions archaïques, il faut recourir à notre intelligence moderne de la violence, toujours imparfaite, certes, mais supérieure à celle du passé. Lorsque il n'est pas mobilisé contre un adversaire bien déterminé, notre appétit de violence s'oriente volontiers vers les individus différents du grand nombre, non seulement les minorités raciales et religieuses, mais les infirmes, les anormaux, les êtres d'exception dans tous les sens du terme, les plus «beaux» comme les plus «laids», les plus obtus comme les plus intelligents, les plus «prophétiques» aussi selon Jésus, tous ceux qu'une différence exceptionnelle signale à l'attention souvent malveillante de la majorité. "Nul n'est prophète en son pays ", dira encore Jésus (voir Mc 6,4 et par.).

Lorsque l'appétit de violence est contrarié, il tend spontanément à se déplacer vers des substituts plus ou moins déplaisants, mais aussi plus ou moins insignifiants, des victimes dont la mort ne risque pas d'envenimer les querelles entre les membres de la communauté. Ce déplacement permet d'assouvir, contre une victime anodine, insignifiante, un appétit de violence dont les effets seraient désastreux s'ils se déchaînaient à l'intérieur de la communauté.

Dans un monde encore très fruste, l'appétit de persécution peut fort bien se rabattre même sur des animaux. Dans le rite judaïque, par exemple, la mauvaise odeur du bouc, son encombrante sexualité, tout ce qui le rend antipathique, a sans doute contribué à faire de lui une victime idéale. Dans notre monde, les traits désagréables du bouc contribuent de toute évidence au succès de l'expression "bouc émissaire». Nous comprenons aisément qu'un animal aussi rébarbatif, même s'il ne "mérite pas d'être persécuté », puisse faire l'objet d'un transfert hostile.

Le troisième sens de "bouc émissaire », le sens psychosocial, permet d'expliquer les deux autres et par conséquent d'attribuer aux religions archaïques une fonction réelle. L'institution religieuse essentielle, c'est le sacrifice. Il reproduit sur des victimes dépourvues d'importance sociale et universellement acceptables les phénomènes de bouc émissaire, dans l'intérêt des communautés humaines perpétuellement menacées par leur propre violence.

Les dictionnaires définissent le sens moderne de "bouc émissaire ", notre troisième sens, comme "dérivé » ou «métaphorique ». J'y vois au contraire le sens premier et fondamental, le phénomène psychosocial 
universellement humain qui permet de comprendre les deux autres sens. Ce troisième sens apparaît très tard et il témoigne d'une compréhension supérieure parmi nous de ce que signifient les rites de bouc émissaire. Pour des raisons que je vais m'efforcer d'éclairer, il y a parmi nous une intelligence grandissante de notre propre violence.

\section{Le phénomène de bouc émissaire}

\subsection{Le bouc émissaire dans les sociétés archaïques}

Les phénomènes de bouc émissaire sont d'autant plus efficaces qu'ils sont moins compris. L'explication transcendantale dont ils faisaient l'objet dans l'univers archaïque est l'origine du religieux. L'unanimité contre le bouc émissaire réconciliait la communauté et passait pour un don de la victime elle-même, perçue comme responsable non seulement des désordres qui justifiaient sa mise à mort, mais du retour à la tranquillité procuré, croyaiton, par le bon vouloir de cette même victime.

Le bouc émissaire spontané semblait tout-puissant pour le bien comme pour le mal. On vénérait en lui une puissance de paix tout en redoutant de sa part, si la discorde se rallumait, un retour de violence. C'est ce que les spécialistes appellent «l'ambivalence» du religieux archaïque. Les boucs émissaires très efficaces étaient finalement divinisés.

Pour renouveler l'effet réconciliateur, les communautés reconnaissantes agissaient toutes de la même manière. Elles s'efforçaient de reproduire l'effet pacificateur en sacrifiant de nouvelles victimes délibérément substituées à la victime originelle. C'est l'émotion suscitée par cette expérience qui importait, c'est elle qui ramenait la paix. C'est l'expérience que les anciens Grecs qualifiaient de cathartique, qui est aussi l'expérience originelle du sacré. Les efforts pour la reproduire artificiellement s'appellent donc des sacrifices.

À force d'être reproduites, les immolations sacrificielles cessaient d'émouvoir les assistants; elles perdaient leur vertu réconciliatrice. Le passage du temps entraînait une "usure» des rites qui aboutissait à une nouvelle crise violente, susceptible de déboucher spontanément, elle aussi, sur un phénomène de bouc émissaire très puissant, un nouveau modèle pour un système sacrificiel efficace parce que renouvelé, jusqu'au moment, bien entendu, où lui aussi perdait cette efficacité initiale et dégénérait en pure routine. 
En dépit de leurs limitations, les sacrifices sanglants fournissaient à l'humanité archaïque son institution essentielle, indispensable, je pense, à l'harmonie des communautés humaines et même, pendant longtemps, à leur survie. Grâce au sacrifice, les cultures archaïques réussissaient à tromper un appétit de violence si intense chez les hommes qu'il suscitait le religieux archaïque dont ils ne pouvaient pas se passer. Sans exutoire rituel, je le répète, l'humanité n'aurait pas survécu.

\subsection{L'universalité du phénomène de bouc émissaire}

Si nous nous examinons attentivement, nous découvrirons en nous-même la présence d'un certain savoir que personne ne nous a jamais enseigné et qui nous renseigne sur la hiérarchie des victimes sacrificielles, identique dans toutes les cultures. Nous voyons tous dans le meurtre d'une créature humaine une action plus émouvante que la mise à mort d'un animal. Et cependant, tuer un animal est une action plus émouvante que d'arracher des légumes pour les manger.

Il y a trois grandes catégories de victimes sacrificielles, l'homme d'abord, l'animal ensuite, le végétal enfin. On peut ajouter une quatrième catégorie, celle des objets matériels, mais à ce niveau-là, on ne parle plus guère de sacrifice. L'institution sacrificielle consiste essentiellement en l'immolation d'êtres vivants. À l'intérieur de ces catégories, il existe des distinctions plus fines qui ne sont pas forcément universelles, mais qui tendent à l'universalité. Le sacrifice d'un homme libre est plus «important» que celui d'un esclave. Les proches sont des victimes plus précieuses que les étrangers. L'être humain le plus proche de nous, c'est nous-même et, dans certaines cultures, le suicide est la forme suprême du sacrifice.

L'existence d'une hiérarchie sacrificielle commune à l'humanité entière contredit le dogme numéro un de l'anthropologie dominante, le relativisme culturel. On veut nous faire croire que les cultures sont complètement étrangères les unes aux autres et complètement fantaisistes. Elles peuvent consister en n'importe quoi. Il est facile de montrer la fausseté de ce dogme. Même aujourd'hui, au-delà de leur suppression presque universelle, les sacrifices se perpétuent sous des formes individuelles et plus ou moins clandestines qui respectent la hiérarchie que je viens de définir. Imaginons le cas d'un employé maltraité par son chef de bureau. Il n'essaie pas de se venger de son persécuteur. Il redoute trop de perdre sa place. Mais lorsqu'il rentre le soir dans sa famille, il est souvent très irrité. Et alors, tout objet fragile qui lui tombe sous la main, une assiette par exemple, il la brise en mille 
morceaux. Si cela ne suffit pas à calmer sa colère, il donnera un coup de pied au chien. Et si cela ne suffit pas encore, il giflera son fils, peut-être même frappera-t-il son épouse...

On ne doit pas voir automatiquement dans ces brutalités la preuve que cet homme est indifférent à sa famille. Il mérite notre réprobation, certes, mais pour comprendre sa conduite, l'indignation ne suffit pas. Il faut recourir à l'idée sacrificielle. Loin de reculer devant la raclée que pourrait lui infliger son chef de bureau, notre employé est probablement plus jeune et plus fort que son supérieur hiérarchique. Il n'a rien à redouter sous le rapport physique. Ce n'est pas la peur qui détermine son apparente lâcheté. S'il craint de perdre sa place, c'est parce qu'il risquerait de ne pas en trouver d'autre. Son premier souci en fin de compte est sa famille. S'il s'en prend à elle plutôt qu'à son persécuteur, c'est pour faire en sorte de ne pas se déchaîner contre lui et, en se faisant obligatoirement renvoyer, priver ladite famille de l'indispensable.

Notre homme ferait mieux de d'abstenir de toute violence, c'est indubitable, mais à tort ou à raison, il se croit incapable de la maîtrise totale de lui-même que la situation idéalement requiert. Il recourt donc à une espèce de compromis avec sa propre violence, à un "calcul sacrificiel» qu'il ne s'avoue pas. Plutôt que d'exposer ses proches à la famine, il préfère les malmener quelque peu. L'idée d'un calcul inconscient est paradoxale et, pourtant, c'est bien ce que nous avons ici, puisque si nous révélions ce calcul, à son auteur, celui-ci soutiendrait mordicus que sa violence n'a rien de sacrificiel et qu'elle est justifiée par le comportement coupable des victimes. Il les a punies, soutiendra-t-il, en raison de quelque faute qu'elles ont réellement commise.

L'optimisme des Lumières s'efforce de ne pas voir ce jeu de la violence sacrificielle et mille autres choses du même genre. Le mythe de la «bonté naturelle » de l'homme, sacro-saint depuis le XVIII siècle, a empêché l'ethnologie de remplir le programme qu'elle s'était tracé, à savoir de découvrir «la nature et la fonction du sacrifice».

Lorsque la vérité est trop déplaisante pour être confrontée, on proclame qu'elle n'existe pas et qu'il n'y a rien à découvrir en dehors des jeux gratuits auxquels les hommes se consacrent lorsqu'ils inventent leurs cultures. En réalité, toutes les sociétés archaïques sont religieuses et sacrificielles. Soutenir comme on le fait encore que le religieux n'a pas de fonction réelle, c'est contredire entre autres choses la théorie darwinienne de l'évolution qui interdit de tenir pour «inutile» une institution aussi universelle 
et aussi durable que le religieux. La théorie du bouc émissaire fondateur confère au sacrifice, et au religieux dans son ensemble, la fonction que l'ancienne anthropologie n'a jamais réussi à découvrir, mais qui n'en est pas moins réelle: il évacue la violence potentiellement fatale que produisent les rapports humains au sein de toutes les communautés.

\section{Le judéo-christianisme et les religions archaïques}

\subsection{Un élément essentiel commun: le phénomène de bouc émissaire}

Et le christianisme? N'est-il qu'une religion archaïque de plus? C'est ce que la vieille anthropologie a toujours affirmé. La preuve que la singularité revendiquée par notre religion n'est qu'un préjugé ethnocentrique, les anthropologues la déduisent des ressemblances assurément remarquables que le christianisme entretient avec les religions archaïques.

Le profil des évangiles ressemble de façon frappante à celui des mythes archaïques. Dans les deux cas, tout commence par une crise aiguë de la communauté entière, une crise qui menace la survie de la communauté. Dans les deux cas, cette crise culmine dans le drame d'une victime unique qui se fait massacrer par la collectivité unanime. Dans les mythes, cette crise se présente sous des formes très diverses. Dans le cas d'CEdipe, c'est une peste qui ravage la ville de Thèbes. Il peut s'agir également d'une catastrophe naturelle: inondation, sécheresse, tremblement de terre... Ou encore d'un monstre qui réclame toujours plus de victimes à dévorer, etc. Dans les évangiles, cette même crise est là et, pour une fois, elle est indubitablement réelle, historiquement certaine. C'est l'étranglement progressif du petit état juif par l'occupant romain. Tout finira par la destruction de Jérusalem et, finalement, de la communauté juive entière.

Jésus est crucifié par l'autorité impériale, d'accord avec les autorités religieuses, appuyées par une foule avide de mise à mort. Tout ce mondelà est en appétit de bouc émissaire. Et, exactement comme dans les mythes fondateurs, la victime, après sa mort, révèle sa propre divinité. Ce parcours est rituellement commémoré et reproduit dans un rite que les chrétiens euxmêmes qualifient de sacrificiel. C'est l'eucharistie, qui n'a plus rien de violent, certes, mais qui n'en reproduit pas moins, comme tous les sacrifices, les étapes essentielles du processus remémoré dans les évangiles.

Les analogies sur lesquelles l'anthropologie antichrétienne attire l'attention sont certainement réelles, indubitables. Les anthropologues ont raison 
de les souligner. Mais ont-ils raison de conclure que le christianisme est trop semblable aux mythes pour ne pas en être un?

Dans le contexte de notre réflexion, il est facile de vérifier que Jésus, lui aussi, meurt en qualité de «bouc émissaire ». Tous les incidents de la Passion rendent cette définition plus manifeste encore que dans les mythes. Comme pour prévenir toute illusion à ce sujet, les évangiles sont truffés de formules et de citations bibliques qui rendent ce rôle de victime innocente complètement manifeste alors que dans les mythes, il faut une analyse «soupçonneuse » pour le découvrir.

Quand je dis bouc émissaire à propos de Jésus, j'entends cette expression bien entendu au sens moderne, au sens psychosocial, celui que j'ai défini en dernier lieu et qu'il faut se garder de confondre avec le rite du Lévitique, comme le font toujours les théologiens qui condamnent ma thèse mécaniquement, sans comprendre en quoi elle consiste. L'expression «bouc émissaire » n'apparaît jamais, bien sûr, dans le Nouveau Testament. Il y en a une autre, cependant, qui la remplace très avantageusement, une formule plus touchante et plus respectueuse, mais qui dit explicitement, cette fois, l'innocence de la victime sacrifiée, et c'est l'expression "agneau de Dieu ". Jésus est le bouc émissaire le plus touchant, le plus innocent qui fut jamais.

Les allusions au rôle sacrificiel de l'agneau sont nombreuses dans les évangiles. Dans celui de Jean, par exemple, une phrase attribuée au grandprêtre Caïphe définit la Passion de Jésus par le bénéfice que devrait en retirer le peuple sous le rapport de la violence. Cette phrase énonce le calcul sacrificiel qui justifie la mort de tous les boucs émissaires: «Il vaut mieux qu'un seul homme meure et que le peuple soit sauvé» (Jn 11,50-51). Le sens de cette formule va évidemment au-delà de la signification seulement politique que le grand-prêtre lui attribue. À la différence des mythes qui déroulent le scénario victimaire sans jamais faire la moindre allusion à la nature et à la fonction des victimes de rechange, les évangiles suggèrent tous que Jésus est bouc émissaire au sens moderne, au sens de la victime qui «paie pour tous les autres ", comme l'affirment nos dictionnaires.

Si les boucs émissaires sont plus visibles encore dans les évangiles que dans les mythes, ne sommes-nous pas obligés d'accepter le verdict de l'anthropologie et de considérer toutes les religions dont nous parlons ici comme équivalentes? Il est clair qu'elles sont toutes enracinées dans le phénomène du bouc émissaire, et tout particulièrement le christianisme. Et notre culture moderne toujours plus déchristianisée voit dans le christianisme une religion identique aux autres, mais qui refuse de reconnaître 
cette identité par pur ethnocentrisme; elle refuse d'admettre sa propre banalité. Elle continue à se prétendre seule vraie, unique et incomparable.

Il y a de nos jours des gens qui se disent chrétiens et qui voudraient contraindre le christianisme à se reconnaître lui-même identique à mille autres religions. Ils se mettent au goût du jour. Ils acceptent de bon cœur le verdict de l'anthropologie et reconnaissent que l'observation objective, scientifique, dément les prétentions du christianisme à la singularité absolue. Tout cela, à leurs yeux, c'est le vieux fatras dogmatique et ethnocentrique depuis toujours charrié par le christianisme et qui doit être rejeté. Selon eux, le christianisme traditionnel est bien trop arrogant et «triomphaliste " pour être authentiquement chrétien. Il faut rabattre son caquet et militer pour une vaste démocratie de toutes les croyances. Notre religion ne méritera d'en faire partie que si elle fait amende honorable et renonce à ses illusions de supériorité. Si le christianisme persiste au contraire dans sa vieille arrogance, il méritera la disparition qui l'attend.

Cette attitude est justifiée, semble-t-il, par la constatation que nous venons de faire. Si Jésus est un bouc émissaire comme tous les autres, nous tenons, peut-être, dans cette définition commune, la preuve certaine que ses prétentions à détenir à lui seul la vérité doit être rejetée. Si Jésus, lui aussi, est un bouc émissaire divinisé, qu'est-ce qui peut encore le distinguer tous les autres boucs émissaires divinisés que sont les dieux archaïques?

Rien, semble-t-il, ne peut distinguer Jésus des autres dieux boucs émissaires. Il est même plus bouc émissaire encore que tous les autres. Les évangiles ne se contentent pas de démontrer la chose en déroulant devant nos yeux la vie et la mort du dieu crucifié, mais ils assortissent ce récit de toutes sortes de formules et de déclarations qui précisent et confirment explicitement son statut de bouc émissaire, et cela les religions archaïques ne le font jamais. Pour dégager le bouc émissaire dans le cas des dieux archaïques, il faut analyser les mythes de façon «soupçonneuse». Les dieux archaïques sont bien des boucs émissaires, mais leurs mythes fondateurs ne confirment jamais expressément la chose. Ils n'y font même pas la moindre allusion. Les mythes ne font jamais ce travail pour nous.

\subsection{L'unicité du judéo-christianisme: la réhabilitation des boucs émissaires}

Si toutes les religions sont fondées en dernier ressort sur le même phénomène, le mécanisme de la victime unique collectivement massacrée parce qu'unanimement reconnue comme coupable, comment soutenir, devant les 
preuves innombrables de sa banalité, que le christianisme est unique et incomparable? Loin de se dérober devant ce défi, les chrétiens peuvent le relever sans la moindre crainte. En dépit des apparences, ils n'ont aucune raison de redouter le type d'analyse que tout le monde fait pour réfuter la revendication chrétienne de singularité et de vérité.

Dans les mythes et les rites archaïques, la victime - Cdipe par exemple - passe pour coupable aux yeux non seulement des êtres qui l'entourent, à l'intérieur du texte que nous lisons, mais aussi aux yeux de l'auteur de ce texte, le poète tragique qui ne change jamais rien d'essentiel au mythe interprété par lui. Même chose dans les évangiles, mais seulement à l'intérieur du texte cette fois, et pas complètement. Presque tous les spectateurs de la crucifixion sont d'accord contre Jésus; ils participent à l'emballement contagieux grâce auquel l'innocent apparaît comme coupable. À la différence de ce qui se passe dans les mythes, toutefois, les auteurs du texte, les quatre évangélistes, ne sont pas d'accord sur le sens que les bourreaux de Jésus donnent à sa crucifixion. Le point de vue qu'ils défendent est celui des quelques disciples qui résistent à la contagion mimétique et qui proclament l'innocence du Crucifié. Mais c'est cette infime minorité, cette fois, qui détient la vérité. Il en va déjà de même pour les grands drames bibliques: ils proclament tous l'innocence des victimes de la violence collective, que les mythes tiennent faussement pour coupables avec la foule des lyncheurs. L'histoire de Joseph dit l'innocence du héros persécuté par ses frères; le livre de Job dit l'innocence du héros persécuté par son entourage. Les Chants du Serviteur disent l'innocence du prophète lynché par la foule...

Dans toute la tradition judéo-chrétienne, ce ne sont plus les victimes qui sont coupables, ce sont les persécuteurs. De même que la Passion, les grands drames bibliques contredisent systématiquement les apparences qui résultent des phénomènes de bouc émissaire, à savoir la culpabilité des victimes. Au lieu de se joindre à la persécution universelle, comme le font les mythes, les grands drames bibliques et évangéliques dénoncent les persécuteurs.

Dans la Bible, la réhabilitation des boucs émissaires commence avec le premier meurtre de l'histoire humaine, celui d'Abel. Si nous comparons ce récit au mythe fondateur de Rome, c'est-à-dire au meurtre de Rémus par son frère Romulus, nous voyons sans peine que, si le contenu objectif des deux récits est le même, l'interprétation est tout autre. Dans les deux cas, il s'agit de deux frères, dont l'un des deux, en tuant l'autre, fonde une société appelée à un grand avenir. Tout est identique à une différence près: dans le mythe romain, le frère assassiné, Rémus, passe pour objectivement 
coupable. Sous prétexte qu'il désobéit aux ordres de son rival plus heureux, il doit être condamné. Au lieu de dénoncer le meurtre, le mythe romain, comme tous les mythes, condamne la victime et justifie le meurtrier. En sa qualité de fondateur officiel de la cité, Romulus ne peut pas avoir tort. Celui qui réussit à tuer son frère le premier, en somme, devient le fondateur immaculé, insoupçonnable, et la victime est universellement rejetée et condamnée. Dans l'histoire de Caïn et d'Abel, les faits sont les mêmes et les résultats aussi, puisque le meurtre d'Abel ne fait qu'un avec la fondation de la première culture. Tout est identique sauf le regard du texte sur lui-même, sur la fondation qu'il nous raconte. La Bible voit très bien que les sociétés humaines reposent sur un meurtre fondateur, mais, à la différence des mythes qui acclament le meurtrier et condamnent la victime, la Bible reproche au meurtrier le crime qu'il vient de commettre. Rien de plus étranger à la mythologie que la fameuse question de Dieu à Caïn: "Qu'as-tu fait de ton frère? » (voir Gn 4,9-10)

Les mythes reflètent les illusions de la violence collective et nous invitent à les partager. Le judaïque et le chrétien dénoncent ces mêmes illusions et nous invitent à les répudier. C'est à cette différence décisive que la pensée moderne tout entière, en dépit de ses prétentions à la non-violence, reste complètement aveugle. Le judéo-chrétien est le seul à dénoncer l'illusion de la victime coupable sur laquelle reposent les religions archaïques, l'illusion du bouc émissaire. Celle-ci structure les mythes bien évidemment et c'est ce que l'anthropologie moderne ne parvient pas à repérer.

Le mécanisme du bouc émissaire, c'est la contagion mimétique qui, dans les périodes de trouble peut soudain rassembler une communauté entière contre une victime innocente, et plus personne alors ne reconnaît cette innocence. Dans les mythes, le processus collectif du bouc émissaire structure le texte et par conséquent reste caché. Le résultat est très utile à la société qui se réconcilie contre la victime, mais très mauvais pour la vérité et pour la justice, puisque la victime, en réalité, est innocente. Le mécanisme de bouc émissaire se caractérise essentiellement par son inconscience. Avoir un bouc émissaire, c'est ne pas savoir qu'on l'a, c'est le prendre pour un authentique coupable: c'est ce que font les mythes. Comprendre qu'on a un bouc émissaire, c'est tomber de son propre piédestal, c'est reconnaître sa propre participation à l'injustice que les univers mythiques ne parviennent pas à repérer. Le processus du bouc émissaire ne devient thème que dans la Bible et les évangiles, et c'est là seulement que le mensonge des mythes apparait. 
À la fin des évangiles et dans les Actes, il y a deux conversions spectaculaires, celle de Pierre et celle de Paul, qui découvrent l'un et l'autre qu'ils se sont acharnés contre un innocent, Jésus lui-même. Cette découverte vient en réalité de Jésus lui-même qui l'enseigne à Pierre et à Paul. Sans Jésus, nous ne saurions jamais qu'il y a des boucs émissaires; nous resterions prisonniers de la structure victimaire. Ce sont toujours la Bible et les évangiles qui démystifient la mythologie et transforment le monde dans lequel nous sommes plongés. Si les mythes ne parlent jamais du phénomène de bouc émissaire, c'est parce qu'ils reposent sur lui. Le souci que nous inspirent les victimes innocentes est un phénomène exclusivement biblique et chrétien qui ne s'enracine pas dans notre bonté naturelle, mais dans l'inspiration biblique.

La formule la plus étonnante dans les évangiles est celle qui nous est proposée par Jésus lui-même avant sa Passion. Elle illumine la fonction majeure du bouc émissaire, son rôle non seulement réparateur dans les sociétés humaines, mais proprement fondateur: la pierre rejetée par les bâtisseurs est devenue la pierre d'angle (Mc 12,10 et par.). C'est une citation de Ps 118,22. Juste avant sa Passion, Jésus demande à ses auditeurs de la lui expliquer et ils restent muets (voir Lc 20,17s.). La réponse est en route depuis deux mille ans et voici qu'elle devient manifeste: c'est une définition du bouc émissaire et de son rôle fondateur dans les communautés humaines.

La Bible et les évangiles défendent et réhabilitent les victimes dont les mythes ne voient pas l'innocence, car ils ne parviennent pas à démystifier l'unanimité contre ces victimes, ils ne voient pas la nature purement mimétique de cette unanimité. Ce que Nietzsche ne voit pas, c'est que toutes les victimes religieuses sont des boucs émissaires, autrement dit des innocents confondus avec des coupables. Il ne voit pas que le judéo-chrétien répudie et démystifie les phénomènes de violence collective que lui, Nietzsche, ne peut pas repérer. Il n'a jamais découvert le mécanisme du bouc émissaire.

Les ressemblances entre violences mythiques et violences judéo-chrétiennes sont dues au fait que dans les deux cas, la même contagion violente triomphe. La différence tient au fait que, dans les mythes, elle triomphe à jamais alors que dans le judaïsme et le christianisme, une minorité dissidente se dresse finalement contre le mensonge. Les apôtres, après la Résurrection, finissent par proclamer la vérité. Le mensonge de la foule ivre de violence imbécile, c'est le dionysiaque qui l'incarne. Seuls le judaïsme et le christianisme révèlent la vérité réservée à la véritable élite, l'innocence 
des boucs émissaires. Il faut se rendre à l'évidence: le judaïsme et le christianisme sont aussi uniques et incomparables qu'ils le prétendent. Ils sont seuls à rectifier le mensonge collectif de toute mythologie.

Si les chrétiens n'ont pas découvert ce que je dis là, c'est parce qu'ils ont toujours éludé le problème des ressemblances entre le mythique et l'évangélique. Ils n'ont pas vu que, pour découvrir la supériorité anthropologique de leur religion, il faut examiner sans crainte ses ressemblances avec les mythes. En éludant cette démarche, ils ont renforcé le relativisme qu'ils s'efforcent d'exorciser.

La vraie démystification du paganisme, c'est le judaïsme et le christianisme qui l'accomplissent. Cela Nietzsche ne l'a pas vu. C'est dans les mythes que triomphe la violence imbécile des foules qu'il exècre. C'est dans le judaïsme et le christianisme seulement que cet esprit est battu en brèche par les petites minorités récalcitrantes, seules capables d'opposer la majorité persécutrice.

Pourquoi existe-t-il une tradition religieuse, et une seulement, qui, prenant le contre-pied des foules polarisées contre leurs boucs émissaires, défend l'innocence des boucs émissaires contre la violence aveugle des foules? Les évangiles donnent à cette question une grande force dramatique en montrant que tous les disciples, et plus particulièrement Pierre, succombent d'abord à la contagion violente, rejoignent la foule de la Passion. Et cependant, trois jours après cette lamentable débâcle, ces mêmes disciples font preuve d'un courage à toute épreuve dans la défense de Jésus, dans la dénonciation de ses persécuteurs. Il y a une impossibilité humaine de ce renversement. Même les meilleurs d'entre nous sont incapables de triompher de la contagion violente par leurs propres forces. Le sens le plus ancien du mot Satan, sans doute, c'est l'accusateur mensonger, le calomniateur qui transforme des innocents en faux coupables, en boucs émissaires. La seule sagesse humaine est incapable de réfuter les mensonges de Satan, les accusations mythiques. Pour tenir jusqu'au bout, il faut l'obéissance de Jésus à son Père, son refus d'échapper à la Croix par des moyens qui le rendraient tributaire de Satan au sens où le sont forcément ceux qui vivent dans les sociétés fondées sur la violence injuste.

Les évangiles ont conscience de ceci, et la preuve, c'est le recours de Jésus, dans l'évangile de Jean, à un seul et même mot pour se désigner luimême et pour désigner l'envoi aux disciples de celui qui les rendra capables de surmonter la contagion violente, le Saint-Esprit. Ce mot, c'est «paraclet », parakleitos, si inattendu au premier abord, que saint Jérôme a 
renoncé à le traduire et l'a simplement transposé en latin. Il signifie simplement le défenseur des victimes faussement accusées, l'avocat de la défense. Ô combien révélatrice est cette signification dans le contexte qui est le nôtre. Tout ce que je viens de dire n'a d'autre ambition que de nous aider à comprendre ce terme capital.

Il ne faut pas conclure de ceci que la transcendance proprement religieuse du judaïsme et du christianisme est démontrable. L'analyse anthropologique n'en bouleverse pas moins un paysage religieux gouverné depuis trois siècles par les amalgames du rationalisme occidental. Cette analyse facilite la foi en restituant au christianisme une puissance intellectuelle que les pensées modernes ne repèrent pas.

Ce n'est pas par ethnocentrisme que le judaïsme et le christianisme se prétendent seuls vrais face à la mythologie. Ces prétentions sont justifiées. L'assimilation du judaïsme et du christianisme au religieux archaïque, c'est la colonne vertébrale du nihilisme moderne, qui est sur le point de s'effondrer.

C’est une véritable révolution, je pense, qui se prépare dans notre intelligence du religieux, un événement bien digne de marquer le passage du second au troisième millénaire après la naissance de Jésus-Christ.

\section{Références}

Frazer, J.G. (1890), The Golden Bough, Londres, Macmillan, 2 vol.

Nietzsche, F. (1964), Euvres complètes / édition critique établie par G. Colli et M. Montinari, Milan, Adelphi.

Schwager, R. (1978), Brauchen wir einen Sündenbock?, Munich, Kösel; aussi paru dans une traduction anglaise: Must There Be Scapegoats? San Francisco, Harper and Row, 1987.

\section{Résumé}

Après avoir précisé le sens premier de l'expression polysémique «bouc émissaire ", l'auteur démontre l'universalité du phénomène ainsi désigné, depuis les sociétés archaïques jusqu'aux contemporaines, universalité qui trouve son fondement dans l'existence d'une hiérarchie sacrificielle commune à l'humanité entière. Sur ce fond, l'auteur compare le judéo-christianisme aux religions archaïques, d'une part explicitant l'élément essentiel qu'ils partagent, le phénomène du bouc émissaire, d'autre part s'inscrivant en faux contre l'assimilation de l'expression judéo-chrétienne de ce phénomène à ses présumés parallèles rencontrés dans les 
religions archaïques. Colonne vertébrale du nihilisme moderne, cette assimilation simpliste occulte sur ce chapitre l'unicité du judéo-christianisme, qui seul défend et réhabilite ses boucs émissaires.

\begin{abstract}
After having specified the primary sense of the polysemous expression "scapegoat", the author demonstrates the universality of the phenomenon thus designated, from archaic to contemporary societies, universality which is founded on the existence of a sacrificial hierarchy common to whole humanity. On this ground, the author compares Judeo-Christianity to archaic religions, on the one hand showing the essential element they share, the scapegoat phenomenon, on the other rejecting the assimilation of the Judeo-Christian expression of this phenomenon with its supposedly parallels found in archaic religions. Spinal column of Modern nibilism, this simplistic assimilation occults on that subject the uniqueness of Judeo-Christianity, which alone defends et rehabilitates its scapegoats.
\end{abstract}

